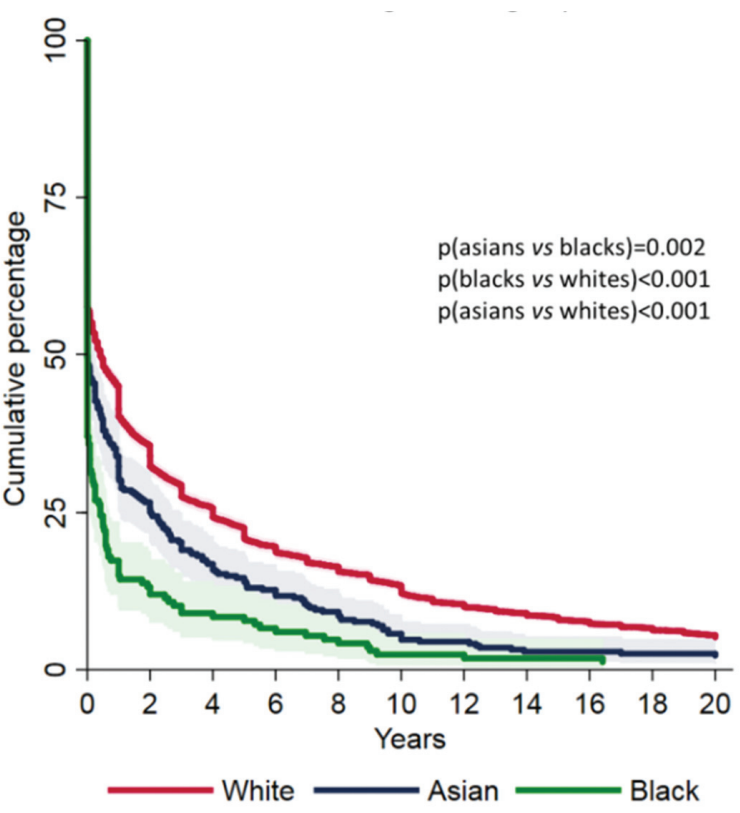

Patients who experienced their first non-RP feature of the disease before the onset of RP were included with a simultaneous onset.

Abstract SAT0474 - Figure 1. Kaplan-Meier curves with $95 \% \mathrm{Cl}$ of the first non-RP feature after RP onset according to racial group.

Patients who experienced their first non-RP feature of the disease before the onset of RP were included with a simultaneous onset.

Conclusions: Several clinical and serological differences were evident between the three racial groups. Asians had high prevalences of Scl-70, $\mathrm{PH}$ and of a reduced FVC. Black patients in contrast had fast disease onset and a high prevalence of diffuse skin involvement.

Disclosure of Interest: None declared

DOI: 10.1136/annrheumdis-2018-eular.3700

\section{SAT0475 ANTI-MDA5 (+) CLINICALLY AMYOPATHIC DERMATOMYOSITIS-ASSOCIATED RAPIDLY PROGRESSIVE INTERSTITIAL LUNG DISEASE: ROLE OF HEMOPERFUSION WITH POLYMYXIN}

A. Selva-O'callaghan ${ }^{1}$, C. Vizcaino ${ }^{2}$, J.C. Ruiz-Rodriguez ${ }^{2}$, E. Trallero-Araguás ${ }^{3}$, N. Ramos ${ }^{4}$, C. Berastegui ${ }^{5}$, A. Gil Vila ${ }^{6}$, M.A. Martinez ${ }^{7}$, X. Solanich ${ }^{8}$, R. Ferrer ${ }^{2}$. ${ }^{1}$ Medicine. Universitat Autonoma de Barcelona, Autoimmune Systemic Diseases Unit. Vall d'Hebron General Hospital; ${ }^{2}$ Critical Care Department, ${ }^{3}$ Rheumatology; ${ }^{4}$ Nephrology; ${ }^{5}$ Pneumology; ${ }^{6}$ Internal Medicine, Vall d'Hebron General Hospital; ${ }^{7}$ Immunology, Sant Pau Hospital; ${ }^{8}$ Internal Medicine, Hospital Universitari de Bellvitge, Barcelona, Spain

Background: Patients with clinically amyopathyc dermatomyositis (CADM) with MDA5 positive autoantibodies may develop a severe pulmonary syndrome with rapidly progressive interstitial lung disease (RP-ILD) with a bad prognosis and high mortality.

Objectives: To analyse the efficacy of a therapeutic protocol that includes hemoperfusion with polymyxin $\mathrm{B}(\mathrm{PMX}-\mathrm{HP})$ in addition to immunosuppressive treatment with tacrolimus and glucocorticoids, plasmapheresis and intravenous immunoglobulin (IVIG).

Methods: We retrospectively analysed 12 (10 male) patients diagnosed with antiMDA5 (+) CADM associated RP-ILD between 1983 and 2018. Anti-MDA5 antibodies were determined by ELISA and confirmed by blot. RP-ILD was defined as a worsening of radiologic interstitial changes with progressive dyspnoea and hypoxemia within 1 month after the onset of respiratory symptoms. From 2014 to 2018, patients (Group 1) were treated with the following protocol: pulses of methylprednisolone (500 mg/day for 3 consecutive days), tacrolimus $2 \mathrm{mg} / 12$ hour, PMX-HP (Toraymyxin 20R, Toray Medical Co., Tokyo, Japan) at a flow of $100 \mathrm{ml}$ h for 3 hour once daily on two successive days, and a plasmapheresis scheme with replacement of 3.5 I of seroalbumin $5 \%$ followed by IVIG infusion $(0.4 \mathrm{mg} / \mathrm{kg})$ during 3 consecutive days and then in alternate days for 7 days more. A comparison was performed with anti-MDA5 (+) CADM associated RP-ILD patients from a historical group (Group 2). $\mathrm{PaO}_{2} / \mathrm{FiO}_{2}(\mathrm{P} / \mathrm{F}$ ) ratio was measured before and after PMX therapy. A composite endpoint that included mortality due to respiratory failure or lung transplantation was defined. Differences between pre- and post-PMX $\mathrm{P} / \mathrm{F}$ values were evaluated using paired sample $t$-test.

Results: Mean (SD) age at diagnosis was 49 (3.5) yrs, with a mean (SD) followup of 13 (3.9) months. Comparison between both Groups showed that 3 out of 6 $(50 \%)$ patients from the Group 1 in comparison with 5 out of $6(83.3 \%)$ from the historical group (Group 2) reached the composite end point. Mean ( $\mathrm{Cl} 95 \%$ ) values of P/F after PMX treatment showed a significantly improvement when compared with the pre-PMX values $\left(240[85-396]\right.$ vs. $\left.125,{ }^{60-189} p=0.038\right)$

Conclusions: Our protocol seems to be useful at some extent in anti-MDA5 (+) CADM associated RP-ILD patients. A transient but significant improvement can be attributed to PMX-HP. Adsorption and elimination of inflammatory cytokines mediators and activated leukocytes, as well as anti-MDA5 antibodies could be the rationale of its efficacy.

Disclosure of Interest: None declared

DOI: 10.1136/annrheumdis-2018-eular.2594

\section{SAT0476 INCIDENCE, PREVALENCE, MORTALITY AND PERFORMANCE OF THE ACR/EULAR 2013 CLASSIFICATION CRITERIA IN PHYSICIAN DIAGNOSED SYSTEMIC SCLEROSIS: RESULTS FROM A POPULATION BASED US COHORT (1980-2016)}

A.S. Sandhu ${ }^{1}$, C.S. Crowson ${ }^{1}$, P.R. Bauer ${ }^{2}$, E.L. Matteson ${ }^{1}$, A. Makol ${ }^{1}$.

${ }^{1}$ Rheumatology, ${ }^{2}$ Pulmonary and Critical Care, MAYO CLINIC, Rochester, USA

Background: Systemic Sclerosis (SSc) is a complex and heterogenous chronic inflammatory disease characterised by widespread fibrosis of the skin and visceral organs, microvascular injury and evidence of immune system activation. Diagnosis can be challenging in the absence of specific laboratory markers or diagnostic criteria.

Objectives: To determine the incidence, prevalence and mortality of physician diagnosed SSc in a population based US cohort and evaluate the performance of the ACR/ ${ }^{\text {EULAR } 2013}$ Classification criteria in comparison to the 1980 ACR criteria in classifying patients with SSc.

Methods: Medical records of patients with a diagnosis or suspicion of SSc in Olmsted County, Minnesota from January 1, 1980 to December 31, 2016 were reviewed to identify incident cases of SSc (defined by physician diagnosis). Prev alent cases of SSc in Olmsted County on January 1, 2015 were also identified. Incidence and prevalence rates were age and sex adjusted to the 2010 US white population. Survival rates were compared with the expected rates in the population of Minnesota. Fulfilment of the 1980 and 2013 classification criteria was ascertained.

Results: A total of 79 incident cases of SSc from 1980 through 2016 and 49 prevalent cases on Jan 1, 2015 were identified. Of these, 71 (90\%) were females, 68 (87\%) were caucasians, age at diagnosis $55.8 \pm 15.9$ years (mean \pm SD). The overall age- and sex- adjusted annual incidence for 1980-2016 was 2.7 (95\% C.I. 2.1 3.3) per 100000 population. The age-adjusted incidence was $4.6(95 \% \mathrm{Cl}$ : 3.55.7) per 100000 for females and $0.6(95 \% \mathrm{Cl}: 0.2-1.1)$ per 100000 for males, with no change in incidence over time. The age- and sex-adjusted prevalence on January 1, 2015 was 47.4 (95\% Cl: 34.1-60.7) per 100000 population.

64 of $79(81 \%)$ patients fulfilled the 2013 classification criteria, while only $48 \%$ fulfilled the 1980 criteria. All but 1 patient that fulfilled the 1980 criteria, also fulfilled the 2013 criteria. All 79 patients had Raynaud's, 38 had cardiopulmonary involvement (pulmonary artery hypertension and/or interstitial lung disease), 33 had digital ulcers/pitting scars, 66 had telangiectasias and 69 had sclerodactyly. 66 patients had limited cutaneous SSc, 11 had diffuse cutaneous SSc and only 2 had Sine scleroderma. 39 patients had a positive autoantibody for SSc: anti-centromere in 29, anti Scl-70 in 8 and anti RNA-polymerase III in 2.

Mortality among SSc patients was significantly higher in comparison to the general population (standardised mortality ratio, 2.24; 95\% C.I., 1.52-3.18). Figure 1 demonstrates survival of 79 Olmsted County residents with incident SSc compared to expected rates from Minnesota lifetables (observed: solid line; expected dashed line). 